\title{
EchoGéo
}

40 | 2017

Ressources urbaines (2)

\section{El uso de la bicicleta en Santiago de Chile ¿es una opción?}

\section{Claudia Arellano Yévenes and Fernando Saavedra Peláez}

\section{(2) OpenEdition \\ 12 Journals}

Electronic version

URL: https://journals.openedition.org/echogeo/14965

DOI: 10.4000/echogeo.14965

ISSN: 1963-1197

\section{Publisher}

Pôle de recherche pour l'organisation et la diffusion de l'information géographique (CNRS UMR 8586)

\section{Electronic reference}

Claudia Arellano Yévenes and Fernando Saavedra Peláez, "El uso de la bicicleta en Santiago de Chile ¿es una opción?", EchoGéo [Online], 40 | 2017, Online since 30 June 2017, connection on 10 August 2021. URL: http://journals.openedition.org/echogeo/14965 ; DOI: https://doi.org/10.4000/echogeo. 14965

This text was automatically generated on 10 August 2021.

EchoGéo est mis à disposition selon les termes de la licence Creative Commons Attribution - Pas d'Utilisation Commerciale - Pas de Modification 4.0 International (CC BY-NC-ND) 


\title{
El uso de la bicicleta en Santiago de Chile ¿es una opción?
}

\author{
Claudia Arellano Yévenes and Fernando Saavedra Peláez
}

\section{Introducción}

1 El acceso a los recursos urbanos ${ }^{1}$ es un factor importante en la producción y mantención de la segregación residencial socioeconómica ${ }^{2}$, como son el acceso a la vivienda, empleo, educación, salud y, donde se centra nuestro estudio, en el acceso al transporte y a la movilidad urbana cotidiana como un recurso de la ciudad (Levy y Dureau, 2002 ; Bertrand, 2010).

2 Este artículo muestra como el uso de la bicicleta se inscribe en un acceso desigual a la movilidad cotidiana, vinculado con el diseño de la red de transporte público que no es igualmente asequible para toda la población de acuerdo a un modelo de ciudad que propicia la segregación espacial. Si "el principal objetivo de la movilidad no reside en el movimiento mismo, sino en el acceso a los recursos del territorio (transporte, servicios públicos, comercio, conocimiento)" Duthilleul (2012, p.572), entonces el acceso desigual a los recursos urbanos mantiene y reproduce la desigualdad social.

Si bien el uso de bicicleta como modo de transporte y forma de acceso a la movilidad tiene una larga historia, esta modalidad así como el ciclista no eran observables, y es a partir de una crisis coyuntural como fue la conflictiva primera fase de implementación del Transantiago que hace visible, una vez más, el problema del sistema de transporte en su conjunto y el de la movilidad cotidiana.

4 El Plan de Transporte Urbano de Santiago (PTUS 2000) planteó el desarrollo de 12 Programas para la modernización del sistema de transporte público. Uno de estos programas fue el Transantiago que comenzó en 2005 y se puso en marcha en 2007, y se convirtió en prioridad y única tarea que arrastró profundos problemas ligados a su organización ${ }^{3}$.

5 El problema del desarrollo urbano vial basado en un modelo de infraestructura con prioridad para el transporte privado y público motorizado del AMS, generó a partir de 
la implementación del Transantiago una crisis en la movilidad cotidiana de las personas, en cuanto a los elevados niveles de congestión vehicular, salvo en las vías de peaje pagado, aumento del tiempo del viaje y los costos de desplazamiento, y sobre todo por la calidad del viaje (Figueroa y Orellana, 2007). Así, "la ejecución de este sistema se convierte en un caso paradigmático por el incumplimiento de los resultados esperados y su negativo impacto social y político" (Corporación Andina de Fomento CAF, 2011, p. 12).

El aumento del ciclismo urbano habría sido parte de una respuesta alternativa al modo de desplazarse de diferentes grupos de la población que se suscitó como una necesidad para un grupo social afectado por la puesta en marcha del Transantiago, sin una previa planificación y prevención de su crecimiento dentro de la fracción modal, ni de las condiciones urbano-viales adecuadas para su masificación (Valenzuela, 2013; Elissegaray, 2009).

El ciclismo urbano en el Área Metropolitana de Santiago de Chile (AMS), nuestra área de estudio conformada por 45 comunas (mapa 1), registró una tasa de crecimiento de 6,8 \% anual de 2001 a 2012 (Ministerio de Transporte MTT, Chile 2015), duplicándose la cantidad de viajes en el periodo (la población creció a una tasa estimada de 1,5\% anual de 2006 a 2012; Armijo y Caviedes, 2008 y 2013), y que a partir de 2007 habría sido una de las respuestas ante los problemas del Transantiago que modificaron la forma de desplazarse de los santiaguinos, alterando la partición modal en detrimento del transporte público (decreció $0,5 \%$ anual) y el aumento de los viajes en modos privados (creció $3 \%$ anual). Además, promovió la movilización de la población que presionó al gobierno local y nacional para transitar hacia un nuevo enfoque de movilidad ${ }^{4}$.

Nuestro análisis del uso de la bicicleta, con base en la Encuesta Origen Destino para la Región Metropolitana EOD 2012 (SECTRA, Chile) ${ }^{5}$, se inscribe dentro del estudio de la movilidad cotidiana no motorizada que corresponde a los desplazamientos realizados por usuarios de los modos caminata y bicicleta, y que además se consideran sustentables ${ }^{6}$. Nuestro foco de interés son los usuarios habituales de la bicicleta, de ahora en adelante los ciclistas ${ }^{7}$. 
Mapa 1 - Área Metropolitana de Santiago de Chile (AMS) por comunas

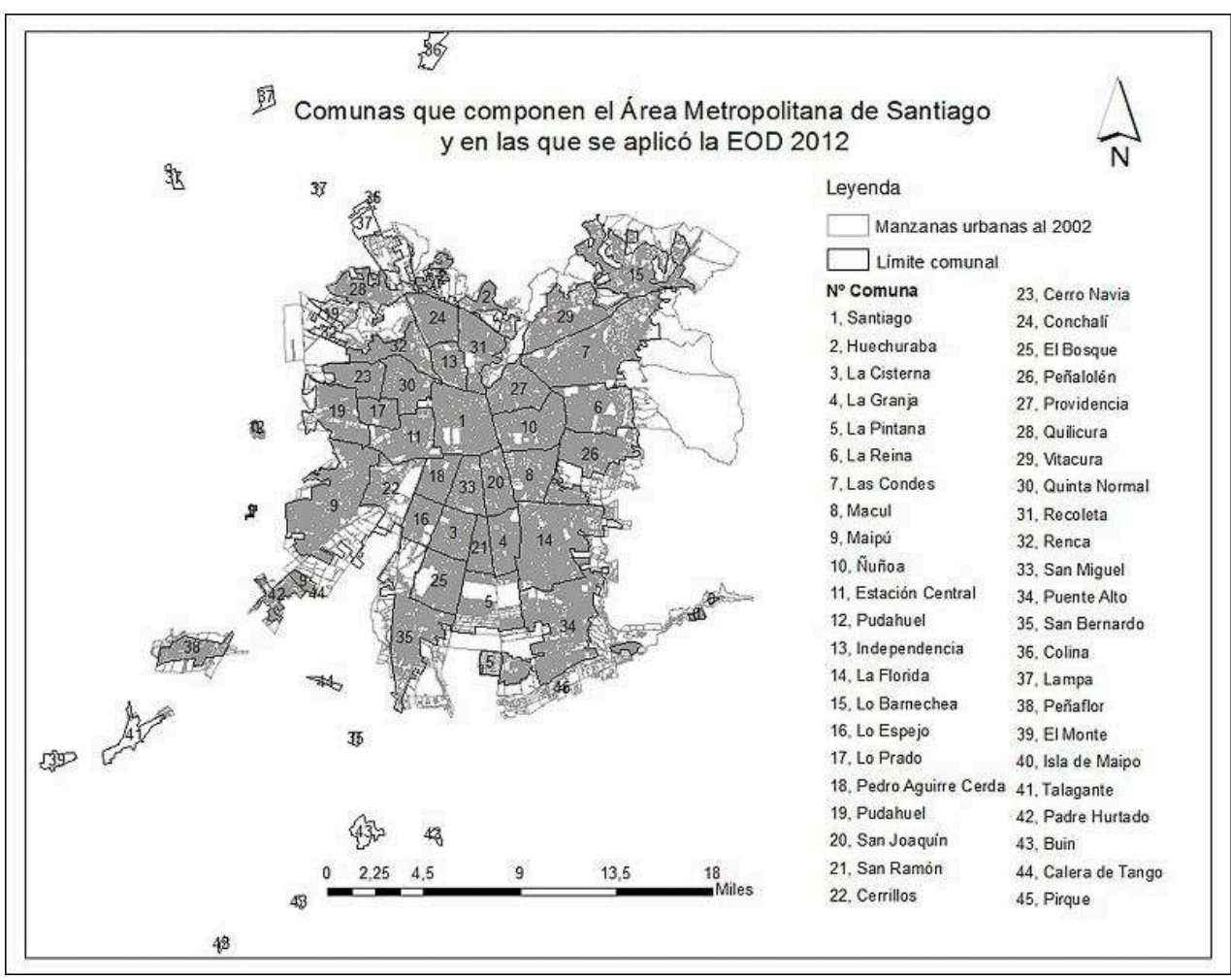

Fuente: elaborado por C. Arellano con base en cartografía del Instituto Nacional de Estadísticas INE.

9 Esta investigación considera que el modelo de ciudad y la estructura urbana y de transporte resultantes establecen las condiciones de la movilidad cotidiana, y en consecuencia del uso de la bicicleta.

El modelo de ciudad mono céntrica y sus posteriores modificaciones a partir de éste, propio de las ciudades latinoamericanas, ha generado una estructura urbana y social diferenciada en el AMS, puesto que su configuración tiende a concentrar a grupos de similares características o estratos socioeconómicos en áreas centrales, que Griffin y Ford (1980) denominan Distrito Central de Negocios. Éste estaría compuesto por aquellas comunas centrales que concentran la mayor parte de los empleos y las actividades desarrolladas por la población en la ciudad (usos mixtos), así como también a la población con mayores recursos, mientras relega a la periferia a los grupos socioeconómicos de menores ingresos, limitando su acceso a oportunidades y actividades.

11 El patrón descrito anteriormente no es del todo seguido en el caso del AMS, puesto existen sectores de medianos y altos ingresos (comunas del noreste) que se distribuyen de manera heterogénea en la ciudad e incluso en la periferia, pero su distribución está moldeada por el modelo de ciudad seguido ${ }^{8}$.

El modelo mono céntrico reproduce la segregación residencial y favorece la desigualdad en la movilidad cotidiana, ya que, según Tokman (2007), quien toma como base los resultados de viajes de la EOD 2001, Santiago sigue siendo una metrópoli preponderantemente mono céntrica.

13 La estructura mono céntrica del AMS ha generado desigualdad en la movilidad de las personas, toda vez que los residentes en áreas centrales y peri centrales tienen menores 
distancias entre el lugar de residencia y de trabajo y de todas las actividades en general, y más opciones de movilidad o más modos de desplazamientos respecto a los habitantes de las periferias, puesto que a pesar de la existencia de una red de transporte urbano de superficie (Transantiago), de líneas de metro, de autopistas pagadas, de ciclo vías y de transportes alternativos como son los taxis colectivos, la red de transporte público por su trazado no favorece a todos por igual.

De acuerdo a lo expresado nos planteamos una serie de preguntas para conocer qué factores están relacionados con el uso de la bicicleta en el AMS y quiénes son los que la utilizan como modo cotidiano de transporte, y así poder establecer relaciones entre las características sociodemográficas de los ciclistas, su residencia y la segregación espacial urbana, contexto donde se inscribe el incremento del uso de la bicicleta.

Formulamos algunas hipótesis iniciales en el sentido que existen factores de eficiencia respecto al tiempo y costo, la accesibilidad a los modos de transporte, las características de la red vial, es decir, factores propios del modelo de ciudad y de transporte implementado que establecen las condiciones para el uso de la bicicleta, y por ello, su uso es para algunos una opción y para otros una necesidad dado sus diferentes lugares de residencia y condiciones sociales. A lo anterior se suman las características demográficas de los ciclistas que condicionan el uso de la bicicleta como medio de transporte para ir al trabajo y/o a estudiar principalmente'.

La EOD 2012 para el AMS se aplicó en 18264 hogares, muestra estadísticamente representativa de la misma. A partir de ésta se elaboró una base de datos con 60054 registros con información demográfica y socioeconómica a nivel individuo, de donde se obtuvieron los viajes realizados en todos los modos (113 591). Esta última base registra los atributos propios de los desplazamientos realizados por cada individuo, como son el número de viajes realizados en el día, el propósito, el modo utilizado, el tipo de vehículo en el caso de los motorizados y bicicleta, el tiempo promedio de cada desplazamiento, la distancia al lugar de trabajo y de estudio, entre otras variables que miden tanto transporte como movilidad.

Con el criterio de dejar sólo a los individuos que viajaron el día de la encuesta en bicicleta como unidad de análisis y observación, se contó con 46263 registros, para obtener finalmente una sub-muestra compuesta por individuos ciclistas auto-declarados como usuarios habituales, que registraron su primer viaje del día en una semana laboral en bicicleta, con lo que se obtuvo una base de 1462 ciclistas, a la que se le pegó las variables a considerar de la base de hogares, con las que es posible conocer el perfil del ciclista. La muestra se trabajó expandida en función de los ponderadores correspondientes.

\section{Antecedentes de la movilidad cotidiana urbana en bicicleta y perspectivas analíticas}

18 Numerosos estudios sobre el uso de la bicicleta se han realizado en diferentes países europeos y de norteamérica tales como los de Albertos y Salom (2011), Furnerss (2010), Kauffman (2008), Miralles-Guash (1999), Pucher, Garrand y Graves (2011), Torres (2003), Sobrino (2008), tanto de tipo cuantitativo como cualitativo, en donde se analiza el ciclismo urbano, la movilidad cotidiana y sus problemas. 
19 En 2011 la CAF y posteriormente el BID comenzaron a realizar diagnósticos específicos de la situación del uso de la bicicleta en América Latina y el Caribe, donde se destaca el AMS como una de las ciudades con mayor número de viajes en bicicleta en términos absolutos (sobre 500000 viajes diarios), pero no en términos proporcionales respecto a los otros modos de la partición modal, que en Latinoamérica no supera el 5,3 \% y que en el AMS estos representaron el 2,1\% en 2001 y el 3,9\% en 2012 dentro del total de los modos de desplazamiento.

20 La Cepal (2010) elaboró un diagnóstico de la situación de la movilidad cotidiana en el AMS de 2006 al 2010, en el cual expresa que es una ciudad altamente motorizada, con un claro déficit de infraestructura para medios de movilidad reducida o no motorizada. Plantea que una política de movilidad exige soluciones para otros sectores de la sociedad (peatones, ciclistas, discapacitados, entre otros) al tiempo que las soluciones técnicas deben tender al co-modalismo en el transporte urbano ${ }^{10}$.

21 En el caso del AMS la Cepal observó medidas que privilegiaban parcialmente a algunos componentes dentro del conjunto, puesto que toda la atención del transporte masivo se concentró en el Transantiago y en la ampliación del Metro, configurando un claro bimodalismo masivo de una manera descoordinada, con una fuerte expansión de la red de autopistas urbanas de pago y una escasa prioridad o total ausencia de modos complementarios y desatención a otras necesidades de la movilidad.

Diverso estudios señalan que la segregación socio espacial pre-existente en el AMS acentúa las inequidades de movilidad entre el transporte motorizado público y privado. ${ }^{11}$ El Transantiago ha mantenido las inequidades y ha tenido un impacto social y político negativo, por tener una deficiente cobertura y calidad en la provisión del servicio (Observatorio de Movilidad Urbana, CAF, 2011).

Investigadores como Ávila Gomide (2003) plantean que el ingreso familiar es una variable que modula y condiciona la forma de desplazarse en Latinoamérica. Por su parte, Jirón (2014) señala que aquellos individuos con menores niveles de educación e ingreso presentan una movilidad menor que aquellos con niveles de educación e ingresos más altos, y que los grupos de menores ingresos se transportan a pie, en bicicletas y transporte público, y los con mayores ingresos se transportan y concentran la propiedad del transporte privado. La Cepal expresa que "el uso de los modos no motorizados de transporte (caminata o bicicleta) y el transporte público son la única opción viable de movilización para los sectores más desposeídos de la sociedad" (CEPAL, Boletín FAL, 2010, Ed n² 289. 8 p.).

24 Un estudio realizado por Elacqua et al. (2011) sobre geografía de las oportunidades en el AMS, revela que existen fuertes diferencias en las oportunidades en general y en particular respecto al acceso a los modos de transporte entre las distintas comunas. Esta situación fue corroborada según el acceso a distintas formas y modos de transporte público y en vía pública en un estudio realizado por Arellano et al. (2013), donde solo una tercera parte de las comunas que forman parte del AMS proveen cobertura de transporte a más del $50 \%$ de la población residente, o más de una opción de acceso al transporte público en un radio de 500 metros, considerando una distancia de caminata a escala de barrio.

Algunos autores señalan los efectos de la estructura metropolitana y social en los movimientos cotidianos y residenciales de la población móvil, donde “(...) también se debe tener presente que, en una sociedad estratificada socialmente, los diferentes 
estratos tienen condiciones también diferentes de accesibilidad a los diversos puntos del espacio urbano" (Ojeda, 2012, p.120). Además, estudios recientes sobre las características del AMS dan cuenta de un modelo de desarrollo urbano mono céntrico medido a través de indicadores de atracción, centralidad, modalidad y cobertura de servicios (Tokman, 2007; Rodríguez, 2008, Escolano y Ortiz, 2005).

Bajo el planteamiento anterior sobre movilidad, la bicicleta es redescubierta y revalorizada no solo en el discurso sino en algunas políticas públicas, como en la Política Nacional de Transporte (PNT 2013, MTT, Chile) y en la Política Nacional de Desarrollo Urbano (2013), en donde se promueve la caminata y la bicicleta para los desplazamientos de mediana distancia, su integración con el transporte público masivo y planes de inversión en infraestructura especializada, y la reglamentación sobre el uso de la bicicleta como modo de transporte y parte de la inter-modalidad.

\section{Cobertura del transporte en el AMS}

Si se observan los mapas 2 y 3 , donde se ve la distribución de las distintas opciones de transporte público en el AMS, se aprecia que las principales redes siguen el patrón de distribución del Metro, las vías principales, al igual que las ciclovías, así como la cobertura del transporte público.

Mapa 2 - Cobertura de las distintas opciones de transporte público en el AMS

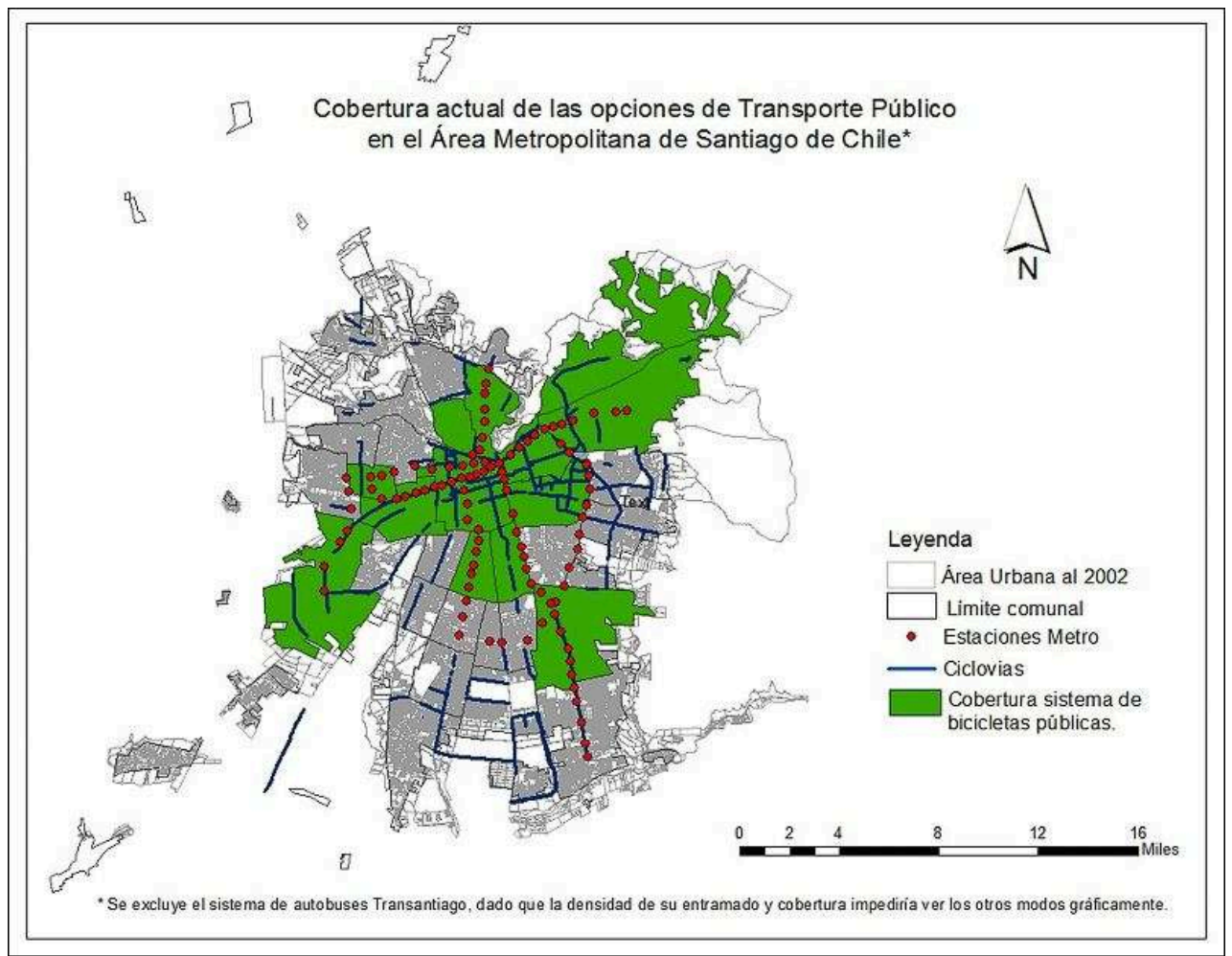

Fuente: elaborado por C. Arellano a partir de diversas bases cartográficas actualizadas al 2016.

La cobertura del sistema de bicicleta pública alcanza a 14 de las 45 comunas del AMS, y recorre las mismas comunas y vías del Metro que favorecen la inter-modalidad, lo cual es una opción más para los residentes cercanos, pero no para la población alejada de 
dichas rutas. Para los que no cuentan con varias opciones para transportarse dado su lugar de residencia y trabajo, el trazado del transporte público y sus condiciones socioeconómicas, este patrón urbano presiona el uso de la bicicleta ante la falta de opciones para esta población, y por otro lado promueve su uso en aquella población donde desplazarse en bicicleta les ahorra tiempo sobre todo.

El sistema de bicicleta pública se planteó desde un inicio del PTUS, y Santiago tiene 137 ciclovías que se extienden por 255 kilómetros de rutas. Éstas se concentran principalmente en comunas de alto poder adquisitivo y mayor motorización (Santiago, Providencia, Ñuñoa y Las Condes) y que serían para los ciclistas donde lo que importa es el ahorro del tiempo de traslado. Sin embargo, hay problemas de diseño y mantención de estas rutas, además de falta de ellas en comunas periféricas (Valencia, 2015).

Mapa 3 - Porcentaje de población de la comuna cubierta por 2 o más modos de transporte en 2012

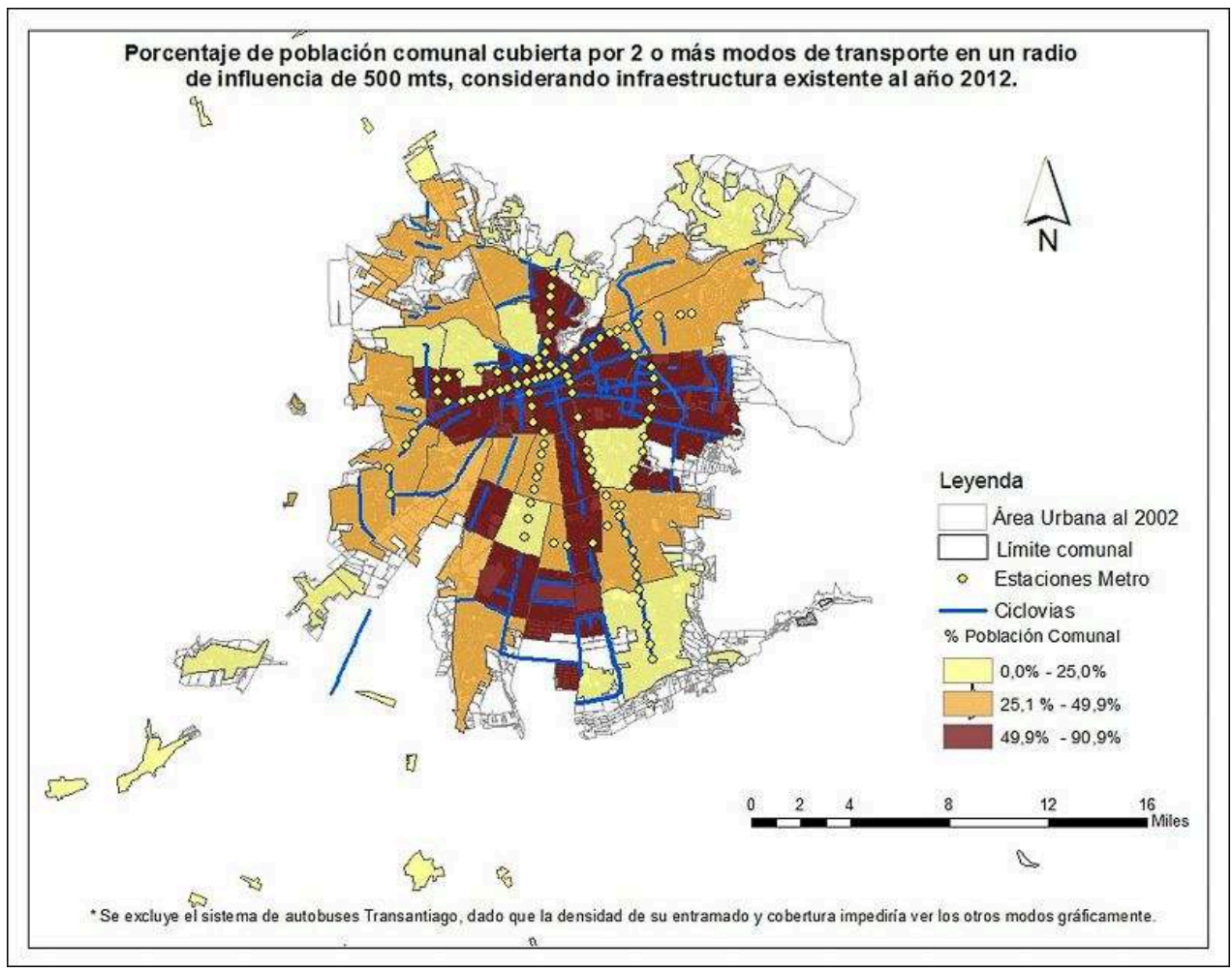

Considerando los paraderos del Transantiago, estaciones del Metro y ciclo vías en un radio de 500 metros.

Fuente: elaborado por C. Arellano a partir de diversas bases cartográficas actualizadas al 2012.

Como se puede observar en los mapas 2 y 3, son las comunas de Santiago y Providencia, y dos corredores: uno noroeste-sureste (comunas de Conchalí, Recoleta, Independencia, Ñuñoa, Macul, La Florida, Puente Alto, con centro en la comuna de Santiago), y otro corredor noreste-suroeste (comunas de Estación Central, Cerrillos, Maipú) los que poseen mayor cobertura de transporte para su población, al disponer a menor distancia de más opciones de transporte público, donde la red del Metro funge como central.

Se puede observar en el mapa 3 como las comunas con más cobertura (zonas oscuras) replican el patrón de la línea del Metro, explicando porque comunas donde reside 
población de bajo nivel socioeconómico como Lo Prado, Peñalolén o La Pintana figuran entre las favorecidas.

\section{El ciclista se hace visible ¿Quiénes son?}

Los datos de la EOD 2012 registraron 747123 viajes en bicicleta en una semana laboral y temporada normal, y el propósito del viaje en bicicleta que más creció fue por trabajo y por estudio (Plan Nosotros Contamos; SECTRA 2012). Otro estudio de 2007 mostró que los horarios de mayor circulación de bicicletas era en las horas punta de la mañana y tarde y en día laboral, muy superiores a los viajes registrados en días festivos ${ }^{12}$.

La bicicleta ha demostrado un uso eficiente en su tiempo de desplazamiento por sobre otras formas de movilidad urbana, al reducir los tiempos de viajes respecto a otros modos motorizados de transporte (individuales y colectivos), además de reducir los costos asociados al viaje.

Según los datos arrojados por las Mediciones de Eficiencia de Modos de Transporte 2008-2014, que midieron el tiempo de viaje de distintos modos (bicicleta, moto, automóvil particular, metro, autobús) en las mismas rutas dentro del AMS durante siete años seguidos y bajo las mismas condiciones, la bicicleta resultó el modo más eficiente, puesto que el mismo recorrido se realizó en promedio en 24 minutos, seguido de la motocicleta con 27 minutos promedio, mientras que el automóvil particular, metro y autobús del Transantiago tomaron en los mismos tramos 37, 41 y 42 minutos, respectivamente ${ }^{13}$.

En los estudios antes mencionados no se ha investigado la relación del lugar de residencia y los viajes por trabajo y estudio (distancia y tiempo) y las características socioeconómicas y demográficas de los usuarios de bicicleta desde una perspectiva de movilidad cotidiana, que es nuestro propósito. Reiteramos que no fue posible incluir otros aspectos fundamentales que están en las decisiones de los individuos de hacer uso de la bicicleta y que obedecen a factores culturales, políticos y a sus representaciones sociales.

36 Los ciclistas registrados fueron 168672 individuos ${ }^{14}$, en su mayoría hombres $(73,4 \%$ y $23,6 \%$ mujeres) y el promedio de edad fue 39 años. Según dos grupos de edad: menores de 15 años y de 15 años o más ${ }^{15}$, podemos diferenciar al grupo en edad laboral y a los menores según su autonomía en el uso de la bicicleta, ya que este usuario es generalmente transportado por otro usuario, y si bien las características del viaje son las mismas, no lo son sus características sociodemográficas, y por ello no se incluyen en este análisis.

El promedio de edad para el grupo de 15 años y más fue de 42 años, y la distribución de probabilidades muestra que a mayor edad el uso de la bicicleta tiende a disminuir.

8 Respecto al nivel educativo, 63 \% de los ciclista tiene al menos un grado de instrucción escolar, 37 \% alcanzó algún grado de enseñanza media, 22 \% con estudios universitarios, muy similar al $21 \%$ que tiene instrucción básica o primaria. Aquellos ciclistas que tienen estudios superiores representaron el $28 \%$, lo que sugiere un nuevo tipo de ciclista, cuya presencia se manifiesta sobre todo a partir del 2007, que ya no se asocia tanto al perfil del ciclista clásico de antaño, que era trabajador con menor educación e ingresos ${ }^{16}$.

Respecto a la condición de actividad que tienen los ciclistas, los que trabajan y reciben un ingreso por ello representaron el $76 \%$; el $18 \%$ declaró que estudia y $7 \%$ que no 
trabaja ni estudia. Para el análisis solo se consideró el propósito del viaje por trabajo y/ o estudio.

Los ciclistas que trabajan, $72 \%$ lo hace como empleado en el sector privado, $20 \%$ son trabajadores por cuenta propia, $3 \%$ empleados de una empresa pública y $1 \%$ como empleado en el servicio doméstico o en la fuerzas armadas. El sector servicios es la actividad principal de las empresas en donde trabajan el 43,9\% de los ciclistas, y $21 \%$ trabaja en la industria, 17,5 en comercio, 4,3 en educación, 2,9 en el sector habitacional, 1,2 en el sector público, al igual que en el de la salud, y el $7.9 \%$ restante en otro tipo de empresas.

41 En cuanto al ingreso mensual del ciclista, $21 \%$ declaró no recibir ingresos de ningún tipo, $31 \%$ percibir entre $\$ 200$ a $\$ 400000$ ( $\$$ es el símbolo de pesos chilenos) que equivale a USD 300 a 600 dólares americanos en 2012, seguido del $22 \%$ que recibe menos de \$200 000 (300 dólares) al mes y que corresponde al "salario mínimo" legal a la fecha de aplicación de la encuesta ${ }^{17}$. Es decir, el $53 \%$ de los ciclistas que perciben ingresos ganan menos de $\$ 400000$ por mes (600 dólares), $8 \%$ recibe entre $\$ 400$ a 800 000, y 3,4 \% recibe $\$ 800$ 000y más. El $14 \%$ no declaró el ingreso.

Los datos señalan que los ciclistas en su mayoría perciben ingresos mensuales cercanos al salario mínimo o apenas superior a éste, lo que contradice la percepción difundida de que el ciclista actual corresponde a una parte de la población con ingresos correspondientes a un profesional. El nivel de ingresos de los ciclistas no sería el factor que diferencia al ciclista clásico del nuevo, sino que es el lugar de residencia que lo ubica en determinado sector social y espacial, aunque ha ocurrido una redistribución de los grupos de medianos ingresos hacia las comunas centrales y peri-centrales (GFK Adimark).

Si consideramos un gasto mínimo en transporte de $\$ 1400$ diarios, ida y vuelta en transporte público, y que el promedio por hogar es de 3 miembros, sería un gasto aproximado de $\$ 84000$ mensuales solo en transporte, es decir, alrededor del $33,6 \%$ de un ingreso mínimo mensual, lo cual es un dato importante que permite argumentar por qué el uso de la bicicleta es una necesidad más que una opción entre otras, sobre todo para el grupo social que reside en la periferia, que serían los ciclistas clásicos que deben usar más de un medio de transporte dado su lugar de residencia y de trabajo, y además no disponen de transporte público cercano.

Los cambios registrados en cuanto a la re-localización de la industria fuera de las ciudades o en sus periferias, así como el proceso de tercerización de las grandes ciudades, se pueden observar en los cambios en las categorías ocupacionales con la disminución de los asalariados industriales (obreros) y aumento de los empleados en el sector servicios y del comercio (Duhau (2003) y Rodríguez (2008), aunque en el caso del AMS $21 \%$ de los ciclistas trabajan en la industria que se localiza preferentemente en la periferia.

\section{El lugar de residencia como factor de desigualdad en la movilidad cotidiana y acceso a los recursos urbanos}

Si analizamos el porcentaje de población ciclista por comuna de origen (mapa 4) y de destino, podemos evidenciar que las comunas de origen de más ciclistas, es decir que cuentan con un porcentaje mayor a la media $(2,1 \%)$, corresponden a dos grupos: los 
que viven en las comunas centrales y peri-centrales de Las Condes (6,7 \%), Providencia (5,8 \%), Nuñoa (5,7 \%) y Santiago (5,3\%), y el otro grupo son los que residen en comunas eminentemente periféricas como Maipú (5,1\%) San Bernardo (4,6\%), Puente Alto $(3,9 \%)$, Quilicura $(3,8 \%)$ y Recoleta $(3,5 \%)$ que son generadoras de más ciclistas respecto al flujo que reciben $(2,2 \%)$.

También son generadoras de ciclistas las comunas de Peñalolén, Lo Espejo, San Joaquín, Cerrillos, Cerro Navia, San Miguel, Renca, Quinta Normal y La Granja, es decir, comunas periféricas y peri centrales, lo que nos revela, por un lado, que la bicicleta es funcional y eficiente como modo de movilidad cotidiana mientras más cerca la residencia del usuario esté del centro de la ciudad, por lo cual los porcentajes de ciclistas son altos en las comunas centrales, y por otro, que en las comunas peri centrales y periféricas que tienen menor disponibilidad de transporte público, la población se ve presionada a usar bicicleta.

Mapa 4 - Porcentaje de Población Ciclista por Comuna de Origen

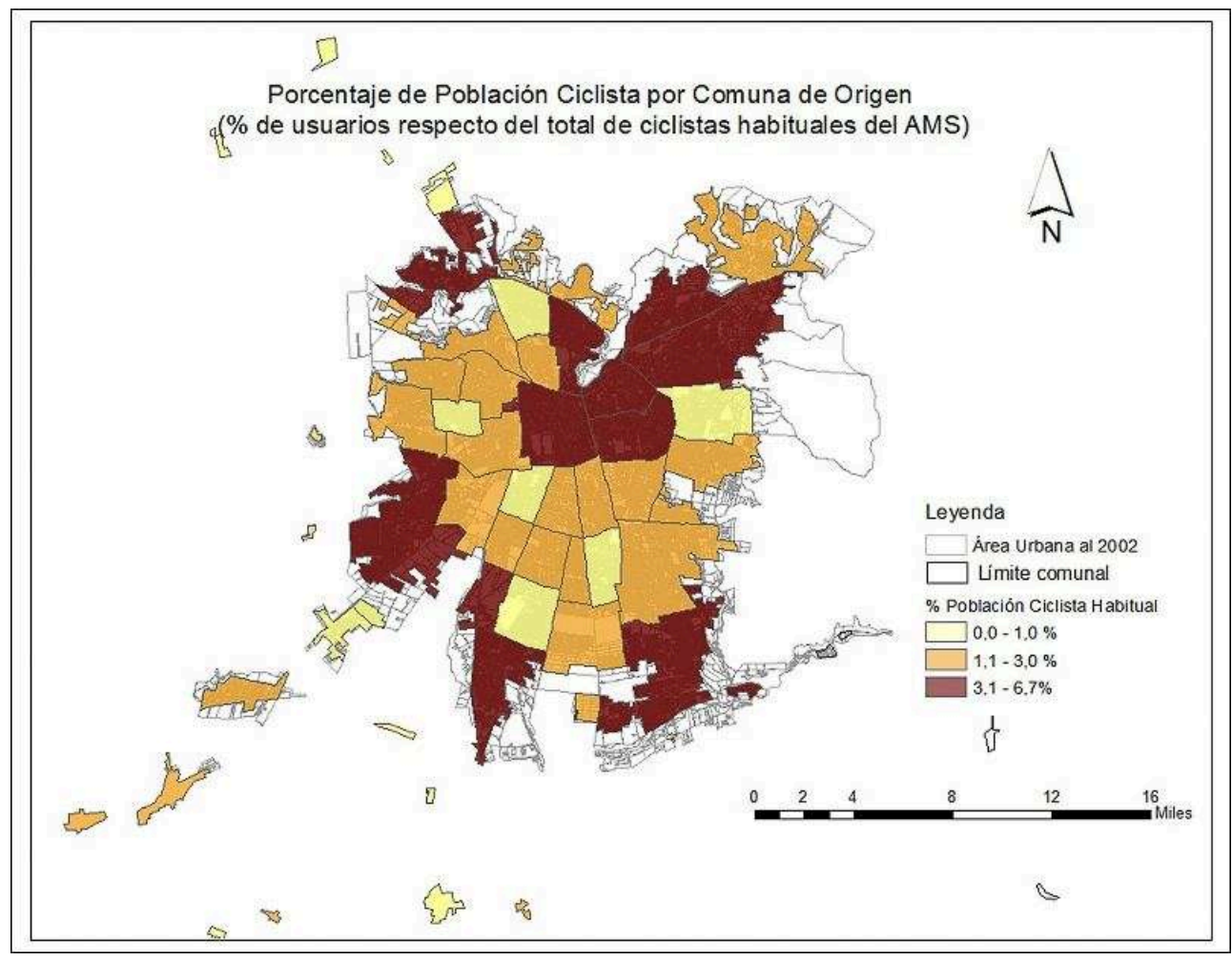

Fuente: elaborado por C. Arellano con base en cartografía del Instituto Nacional de Estadísticas (INE).

Las comunas que reciben mayor número de ciclistas (comunas de destino) son Santiago, Providencia, Ñuñoa y Las Condes, siendo Santiago la que recibe 4 veces más ciclistas que la media de las comunas de destino con un $8 \%$ del total. Si consideramos aquellas comunas que reciben más ciclistas de los que generan o residen en su jurisdicción, se suman algunas comunas peri central como Vitacura, Macul, Huechuraba, Independencia, La Florida y La Cisterna.

Existen otras comunas generadoras de ciclistas localizadas distantes del centro, como Talagante, Peñaflor e Isla de Maipo, en donde los ciclistas residen pero tienden a 
desplazarse a comunas vecinas en la periferia donde se localizan las industrias, que correspondería al tipo que denominamos como ciclistas clásicos.

El $99 \%$ de los ciclistas son propietarios de la bicicleta que usan, y respecto a las condiciones de los viajes, por dónde desplazarse y dónde dejar la bicicleta, la mayoría de los ciclistas no utiliza ciclo vías ( $60 \%$ ), y del resto que lo hace $23 \%$ las usa en parte de su trayecto y solo el $17 \%$ las usa en todo el viaje.

Ante la pregunta del por qué no usan ciclo vías, 95 \% señaló que no existían en su ruta, $3 \%$ porque el acceso a ella no es directo o debe pasar por calles sin ciclo vía para llegar a ellas, y $1 \%$ señaló que su diseño no es atractivo o seguro y prefiere usar la calle. Así, tanto el uso de ciclo vías como el lugar donde dejar la bicicleta indica que las condiciones de la estructura urbana para los ciclistas son insuficientes, hay inseguridad en los viajes y riesgo por estacionarla en la vía pública.

51 Para Hurtubia y Muñoz (2016) el que se hayan duplicado los viajes en bicicleta de 2001 a 2012 se relaciona con la construcción de ciclovías y por el incremento del uso en comunas de altos ingresos, donde muchos de sus habitantes se dieron cuenta de que para distancias menores a $5 \mathrm{~km}$ la bicicleta es la mejor alternativa (nuevos ciclistas).

Sin embargo, los niveles promedio de ingresos declarados por los ciclistas (EOD 2012) son alrededor de dos salarios mínimos (menos de $\$ 400000$ ), muy por debajo del promedio de estas comunas de altos ingresos que es de 2 millones de pesos chilenos y que corresponde a los niveles socioeconómicos $\mathrm{ABC}$. Pero también en estas comunas reside clase media del nivel C2 cuyo promedio de ingresos es de $\$ 600000$ pesos, lo que estaría señalando una mayor heterogeneidad social en estas comunas que se han densificado según GFK Adimark (2013), quien formula que el segmento C2 se caracteriza por un comportamiento similar al $\mathrm{ABC} 1$, aunque con una tendencia a vivir en sectores más próximos al centro y con ello a las oportunidades que ofrece la ciudad. La densificación de comunas como Santiago, San Miguel, Ñuñoa y Providencia son características de este fenómeno.

\section{Conclusiones}

Los ciclistas nuevos serían los residentes en comunas centrales o peri-centrales que cuentan con más opciones de transporte y conjugan tiempo y distancia, lo que les motiva al uso de la bicicleta para ir al trabajo, en tanto los ciclistas clásicos residentes en la periferia que trabajan en las industrias cercanas o deben recorrer distancias mayores y más tiempo en recorrerlas cuando se dirigen al centro, no cuentan con otras posibilidades de transporte y se ven compelidos a usar bicicleta.

Si se consideran las características sociodemográficas y las referentes a los viajes, un tipo de ciclistas serían aquellos con un perfil cercano al ciclista clásico, donde el $44 \%$ de los viajes son con motivo laboral, con jornada completa en empresas privadas, principalmente servicios o industria, son asalariados y conforme a sus ingresos se trataría de un grupo social medio y bajo, que tiene educación secundaria, y que residen principalmente en comunas periféricas.

55 El otro tipo correspondería al nuevo ciclista, que también va al trabajo en bicicleta, pero que reside en comunas centrales o peri-centrales más cercanas a su lugar de trabajo (distancias menores a 5 kilómetros), trabajan como empleados o cuenta propia en el 
comercio, un relativo mayor nivel educativo, pero también con niveles de ingreso de clase media, y por ello su diferenciación no es por el nivel de ingresos.

La segregación territorial y el trazado de la red de transporte propician la diferenciación en la movilidad cotidiana en general y entre los ciclistas en particular. Además favorece la invisibilidad del ciclista clásico que se desplaza y reside en la periferia. Los distintos tipos de perfiles tienden a moverse y desplazarse entre zonas con similares características tanto de su urbe como de su población, replicando la estructura social en la movilidad, de manera que los desplazamientos de los ciclistas son en comunas y sectores de similares características, evitando así el encuentro del otro tipo $^{18}$.

57 En lo que respecta al modelo urbano y las políticas públicas se observa que persisten serios problemas de una verdadera ciclo-inclusión, en donde se considere al individuo y no solo el viaje, como se postula, relacionado con las pocas alternativas que tiene la población residente en donde no hay buena cobertura del transporte público (periferia) $\mathrm{y}$ donde las tarifas son muy elevadas para los bajos ingresos que reciben numerosas familias.

Los resultados del análisis de la información muestran que para el 2012 los necesarios cambios para una movilidad cotidiana eficiente aún no se manifiestan, y tampoco se observa una disminución de las desigualdades territoriales y la segregación espacial, donde el uso de la bicicleta se refleja en estos dos tipos de usuarios, congruente con el modelo urbano implementado.

59 También hay que considerar que el AMS está experimentando un reacomodo de la población que busca instalarse en las proximidades de los medios de transporte colectivos (Metro y Transantiago) para disminuir los tiempos de traslado, lo cual es posible por una cierta oferta de viviendas producto de la re-densificación de ciertas comunas donde puede acceder población de sectores medios, y que la construcción de ciclovías en estas zonas hace más atractivo el uso de la bicicleta para ellos (GFK Adimark, 2013).

60 Si bien la ciclo-inclusión ayuda y favorece una movilidad menos contaminante y reduce la congestión vehicular, ésta tiene limitaciones ligadas al modelo de transporte que aún favorece el uso de los motorizados, tanto particulares-privados como públicos, y dadas las características de los ciclistas en cuanto a su lugar de residencia y trabajo, el acceso a los diferentes modos de transportes dentro de una ciudad segregada espacialmente, condiciona las opciones. Por ello, mantener el uso de la bicicleta y poder aumentarlo está condicionado al cambio del modelo urbano más que a políticas sectoriales, además del cambio en las representaciones sociales que implica, tema no tratado en este artículo. 


\section{BIBLIOGRAPHY}

Albertos J., Salom, J., 2011. Determinantes de la movilidad diaria por razón de trabajo en las áreas metropolitanas españolas. Revista Población y Espacios Urbanos, Universidad de Barcelona, España. Cuadernos de geografía, n 90, p. 227-244.

Arellano C. et al., 2013. Geografía de las Oportunidades: Análisis georreferenciado sobre la Disponibilidad de Servicios que Impactan la Calidad de Vida. Instituto de Política Pública, Universidad Diego Portales, Chile. 123 p.

Ávila Gomide A. de, 2003. Transporte urbano e inclusión social: elementos para políticas públicas. Ministerio Planeación, Brasilia. Instituto de Pesquisa Econômica Aplicada - ipea, 37 p.

Banco Interamericano de Desarrollo BID, 2014. Biciudades 2014: Atlas dinámico del ciclismo urbano en América Latina y el Caribe. $37 \mathrm{p}$.

Banco Interamericano de Desarrollo BID, 2015. Ciclo-inclusión en América Latina y el Caribe: Guía para impulsar el uso de la bicicleta. IMCO, $34 \mathrm{p}$.

Bertrand M., 2010. Introduction. Revue Tiers Monde, 1/2010, n² 201, p. 7-23.

Buehler R., Pucher J., 2012. City Cycling Urban and Industrial Environments. Editado por John Pucher and Ralph Buehler. The MIT Press, Cambridge, Massachusetts, Institute of Tecnology; London, England. 365 p.

CEPAL, 2009-2010. División de Recursos Naturales e Infraestructura. Serie Boletín FAL, n² 289, p. 8.

Comisión Asesora Presidencial Pro Movilidad Urbana, 2014. Problemas de Movilidad Urbana: estrategia y medidas para su mitigación. Gobierno de Chile, Santiago, Chile, 202 p.

Comisión Económica para América Latina CEPAL, 2010. Boletín FAL. División de recursos Naturales e Infraestructura, 8 p.

Comisión Económica para América Latina CEPAL, 2014. Panorama Social de América Latina 2014. Oficina de Naciones Unidas, ONU: LC/G.2635-P., 298 p.

Corporación Andina de Fomento (CAF), 2010. Análisis de la movilidad urbana: Espacio, medio ambiente y equidad. BID, Bogotá, Colombia. $117 \mathrm{p}$.

Corporación Andina de Fomento (CAF), 2011. Observatorio de Movilidad Urbana. Desarrollo y movilidad en América Latina. CAF, Bogotá, Colombia, 315 p.

Delaunay D., 2010. Mobilités, ségrégations résidentielles et bonus démographique dans la zone metropolitaine de Santiago du Chili. Revue Tiers Monde, 1/2010, n² 201, p. 65-85.

Dirección General de Tránsito DGT, 2010. Barómetro Anual De La Bicicleta: España. Dirección General de Tránsito, $231 \mathrm{p}$.

Duhau E., 2003. División social del espacio metropolitano y movilidad residencial. Papeles de Población, vol. 9, n 36, Universidad Autónoma del Estado de México. p. 161-210. http:// redalyc.uaemex.mx

Duhau E., Giglia A., 2003. Las reglas del desorden: habitar la metrópoli. México, Siglo XXI Editores/ UAM-Azcapotzalco, 508 p. http://www.scielo.org.ve/scielo.php?

script=sci_arttext\&pid=S1012-25082012000100010\&lng=es\&nrm=iso 
Dupont V., Dureau F., Lelièvre E., Lévy J.P., Lulle T., 2000. Introduction générale. In Dureau et al. (ed.), Métropoles en mouvement : une comparaison internationale. Paris, Anthropos-IRD, Coll. Villes, p. 3-12.

Dureau F., Barbary O., Lulle T., 2007. Dinámicas metropolitanas de poblamiento y segregación. In Dureau F., Barbary O., Gouëset V., Pissoat O. (coord.), Ciudades y sociedades en mutación. Lecturas cruzadas sobre Colombia. Bogotá, Universidad Externado de Colombia, p. 161-235.

Duthilleul A., 2012. Circuler: quand nos mouvements façonnent la ville. Editions Alternatives; París, 208 p.

Elacqua G. et al., 2011. Geografía de las Oportunidades Educacionales en Chile. Análisis Geo-Referencial que identifica barrios de bajas oportunidades en educación. Instituto de Políticas Públicas, Universidad Diego Portales, Chile. 16 p.

Elissegaray P., 2009. Uso de la Bicicleta en la Región Metropolitana: Diagnóstico Perspectivas y Desafíos. Universidad de Chile, Facultad de Sociología, Departamento de Sociología, mémoire d'étudiant, $91 \mathrm{p}$.

Escolano S., Ortiz J., 2005. La formación de un modelo policéntrico de la actividad comercial en el Gran Santiago. Revista de Geografia Norte Grande, n 34, p. 53-64.

Figueroa O., Orellana A., 2007. TranSantiago: gobernabilidad e institucionalidad. Revista Eure, vol. XXXIII, n 100, Pontificia Universidad Católica de Chile; Santiago, Chile. p. 165-171.

GFK Adimark, 2013. Estudio Chile 3D Cambios: Marcas y Consumo 2002 y 2013. Chile.

Gobierno Regional Metropolitano de Santiago, 2009. Ciudad Viva. Plan Maestro de Ciclorutas para Santiago, Chile.

Gouëset V. et al., 2015. Recorrer la metrópoli: prácticas de movilidad cotidiana y desigualdades socio-territoriales. En Dureau F., Lulle T. y Contreras Y. (editores), Movilidades y Cambio Urbano: Bogotá, Santiago y Sao Paulo. Universidad Externado de Colombia, Cap.8; p. 303-344.

Griffin E., Ford L., 1980. A Model of Latin American City Structure. Geographical Review, vol. 70, $\mathrm{n}^{\circ}$ 4, American Geographical Society, p. 397-422.

Gutiérrez A., 2012. ¿Qué es la Movilidad? Elementos para (re) construir las definiciones básicas del campo del transporte. Revista Bitácora, vol. 2, n 21, Universidad Nacional de Colombia, Octubre, $74 \mathrm{p}$.

Hurtubia R., Muñoz J. C., 2016. El nuevo Chile también va en bici. Voces la Tercera, Chile, 19 abril de 2016.

Iceland J., Wilkes R., 2006. Does socioeconomic status matter? Race, class, and residential segregation. Social Problems, vol. 53, n 2, Issue 2.

Instituto Nacional de Estadísticas, Chile, 2001. Encuesta Origen Destino (EOD). Instituto Nacional de Estadísticas, Chile, 2002. XVII Censo de Población y Vivienda.

Jouffe Y., 2011. Las clases territoriales entre movilidad metropolitana y repliegue barrial $\iota^{\text {tienen }}$ los pobladores pobres una movilidad urbana de clase? Revista Transporte y Territorio, $\mathrm{n}^{\circ} 4$, UBA, Argentina. p. 84-117.

Kauffman V., 2008. Les paradoxes de la mobilité, bouger, s'enraciner. Revista Méditerranée. Migrations et territoires de la mobilité en Méditerranée, $115 \mathrm{p}$.

Levy J.P., Dureau F. 2002. L'accès à la ville. Les mobilités spatiales en question. París, Belín, 411 p. 
Ministerio de Transportes y Telecomunicaciones (MTT), 2013. Plan Maestro de Transporte Santiago 2025, Chile.

Ministerio de Transportes y Telecomunicaciones (MTT), 2015. Nota de prensa 19 marzo 2015 http:// www.mtt.gob.cl/archivos/10194

Miralles-Guach C., 1999. Movilidad en la ciudad: la irrupción del transporte privado en la ciudad. Ecología Política, Ed. Icaria, Barcelona, 174 p.

Miralles-Guasch C., 2000. Movilidad sostenible: innovaciones conceptuales. Elementos del Debate Territorial, n 10, UAB, España. p. 193-216.

Miralles-Guash C., Marquet, O., 2009. Ciudad Compacta, la otra cara de la ciudad sostenible. Revista Ambienta, Departamento de Geografía, UAB, España, nº 100, p. 16-27.

Ojeda M., 2012. El Modelo del área metropolitana de Santiago ¿Ciudades mono o Policéntricas? Impactos de la Configuración Urbana en la Población; Facultad de Arquitectura, Universidad de Chile, mémoire d'étudiant. $171 \mathrm{p}$.

Olavarría M., 2013. De la formulación a la implementación del Transantiago: Análisis del proceso político de una política pública. Revista Gestión y Política Pública, vol. 22, $\mathrm{n}^{\circ} 2,2^{\mathrm{e}}$ semestre, p. 355-400.

Programa de Naciones Unidas para el Desarrollo PNUD, 2008. Plan de Seguimiento del Programa de Fomento al Uso de la Bicicleta en las comunas de Providencia, Santiago y Ñuñoa.

Pucher J., 2011. Cycling labor in 90 large US cities: New evidence on the role of bike paths and bike lanes. Transporte Trimestral 98-1, http://www.ibike.org/library/statistics-data.htm

Rodríguez J., 2008. Dinámica sociodemográfica metropolitana y segregación residencial: ¿qué aporta la CASEN 2006? Revista de Geografía Norte Grande, 41, p. 81-102.

SECTRA Secretaría de Transporte, 2005. Plan de Transporte Urbano para Santiago.

SECTRA Secretaría de Transporte, 2012. Encuesta Origen Destino Santiago, Chile 2012.

SECTRA Secretaría de Transporte, 2013. Análisis Normativo de la Bicicleta: Diagnóstico de la Bicicleta en Chile. $302 \mathrm{p}$.

SECTRA Secretaría de Transporte 2015. Informe Difusión de la Encuesta Origen Destino para la región Metropolitana 2012, Chile.

Sobrino J. 2008. Medición de la movilidad cotidiana a través del censo de población: la experiencia en México. El Colegio de México, Presentación, 12 p.

Tokman M., Rodríguez J., 2007. Chile y su Esfuerzo Innovador: Avances y Desafíos. En Cisternas L., Morales P., Ciencia y Tecnología en las Regiones de Chile, junio 2007, p. 15-42.

Torres R., 2003. La bicicleta ¿una alternativa real de transporte urbano? El caso de VitoriaGasteiz. Ciudades para un Futuro más Sostenible, Boletín CF+S 28, Centro Estudios Ambientales del Ayuntamiento de Vitoria-Gasteiz, España. 16 p. http://habitat.aq.upm.es/boletin/n28/artor.html Universidad Católica de Chile, Departamento de Ingeniería y Logística de Transporte, 2014. Mediciones de Eficiencia de Modos de Transporte 2008-2014. Santiago de Chile.

Valencia M., 2015. Santiago tiene 137 ciclovías, y un tercio de ellas no se encuentran en buenas condiciones. El Mercurio, Chile, 12 agosto 2015. 
Valenzuela A. 2013. El boom del ciclismo urbano. El rol del desarrollo urbano y las políticas públicas. Estudios Urbanos y Territoriales, P. Universidad Católica de Chile, mémoire d'étudiant, 31 mayo 2013, 134 p.

\section{NOTES}

1. La noción de recursos urbanos parte de una definición amplia que comprende no solamente los recursos físicos (accesibilidad, redes urbanas, servicios, transporte, mercados de trabajo, etc.) sino también los recursos específicos que presenta un lugar para un individuo o familia y las redes de inter-conocimiento (Proyecto RESUM, UMR-PRODIG, Universidad de París 1; Ribardiere y Michel., 2014; comunicación interna).

2. El concepto de segregación residencial socioeconómica se refiere a cómo los grupos poblacionales están distribuidos de manera desigual a través del espacio urbano. Es el grado en que diversos grupos (determinados por su ingreso, religión o estatus migratorio, entre otros) comparten áreas residenciales o barrios (Iceland y Wilkes, 2006, p. 249).

3. Dentro de los principales cambios que hubo entre el sistema antiguo y el PTUS estaban el diseño de buses nuevos, nuevos recorridos, paraderos diferidos, uso de tarjeta de prepago en vez de dinero, combinación de trasbordo con el Metro que ahora pasaba a ser público, corredores especiales y exclusivos para los buses troncales (Figueroa y Orellana, 2007).

4. El estudio de la movilidad cotidiana se enmarca dentro del concepto amplio de movilidad urbana, definida por Miralles-Guash (2000) como la suma de los desplazamientos realizados por la población de forma recurrente para acceder a bienes y servicios en un territorio determinado.

5. Se han realizado dos Encuestas Origen Destino: EOD 2001 (Instituto Nacional de Estadísticas, Chile (INE), y EOD 2012 (INE) cuya información y acceso a los datos fueron liberados en 2015, producto del ajuste realizado al Censo de 2012 (INE).

6. La movilidad sustentable privilegia los modos que reducen los impactos socio-ambientales, disminuyen el consumo de energía, disminuyen el tiempo de los desplazamientos, hacen más eficientes los viajes y mejoran la calidad de vida urbana. Así, los desplazamientos a píe, bicicleta y el transporte público son favorecidos (Torres, 2003, p. 1; Gutiérrez, 2012, p. 64).

7. Los usuarios habituales de la bicicleta que nombramos como ciclistas, son aquellos que se autodeclararon como tales en la EOD 2012 (SECTRA).

8. GFK Adimark, Estudio Chile 3D Cambios: Marcas y Consumo 2002 y 2013.

9. Dada la fuente y la información existente, no se aborda en este trabajo el tema de las representaciones sociales respecto al uso de la bicicleta, las cuales son diferentes según sexo, edad, pertenencia social, entre otras, que influyen en la toma de decisiones al respecto.

10. El co-modalismo es la planeación y combinación de todas las alternativas técnicas que complementan la debida atención de las necesidades de movilidad, con el fin de que cada modo participe en los viajes de la manera en que es más eficiente socialmente, maximizando la eficiencia del total de cada viaje (Boletín FAL, Ed. 268, Cepal, 2010).

11. Ojeda, 2012; Delaunay, 2010; Gouëset V. et al., 2015 en Dureau, Lulle y Contreras Y. (editores); Jouffe 2011.

12. Plan de Seguimiento del Programa de Fomento al Uso de la Bicicleta en las comunas de Providencia, Santiago y Ñuñoa. PNUD, 2008.

13. Universidad Católica de Chile, Departamento de Ingeniería y Logística de Transporte; Mediciones de Eficiencia de Modos de Transporte 2008-2014, Santiago 2014.

14. Es el número de ciclistas de la muestra ponderada (según el factor de expansión).

15. El AMS tenía una población estimada de 6,8 millones de habitantes en su porción urbana en 2013. Por grupos de edad alrededor del $23,5 \%$ eran menores de 15 años, $55 \%$ de 15 a 49 años y 
$21,5 \%$ de 50 y más. Un poco más de la mitad eran mujeres (en el país alrededor del $21 \%$ tenía menos de 15 años, 59,5 \% de 15 a 54 años y 19,8 \% de 50 años y más).

16. A nivel nacional el $61,48 \%$ de la población tiene al menos un grado de instrucción escolar, $33,8 \%$ algún grado de enseñanza media y $15,4 \%$ con estudios universitarios.

17. El sueldo mínimo a la fecha de aplicación de la encuesta era de $\$ 200000$ mensuales, y el dólar estaba a $\$ 600$, un poco más de 300 dólares por mes.

18. No es usual que entre ambos perfiles haya un encuentro móvil, salvo en aquellas ocasiones donde el espacio público se cierra especialmente para modos no motorizados en los domingos, donde dada la extensión del cierre de calles, se entrecruzan prácticamente todos los perfiles en el mismo espacio.

\section{ABSTRACTS}

El acceso al transporte y a la movilidad cotidiana constituye uno de los factores importantes de los recursos urbanos de una ciudad, y este acceso es desigual en Santiago, producto de la segregación residencial y socioeconómica de su población y una red de transporte público que no es de fácil acceso para ciertos grupos de población. Este artículo plantea que el uso de la bicicleta como modo de transporte muestra la desigualdad en las condiciones de los desplazamientos, donde para un grupo de la población es una opción y para otro es por falta de alternativas para transportarse, y el ingreso no es el factor diferenciador, sino el lugar de residencia respecto al del trabajo. Mediante la caracterización sociodemográfica de los usuarios de bicicleta, de los viajes y del lugar de residencia y destino se analiza su inclusión en la movilidad cotidiana.

\section{INDEX}

Palabras claves: movilidad cotidiana, desigualdad, acceso a transporte, uso bicicleta, segregación residencial.

Subjects: Sur le Champ - Sur le Terrain

\section{AUTHORS}

\section{CLAUDIA ARELLANO YÉVENES}

Claudia Arellano Yévenes, claudia.arellano@flacso.edu.mx, Maestra en Población y Desarrollo. Facultad Latinoamericana de Ciencias Sociales sede México.

\section{FERNANDO SAAVEDRA PELÁEZ}

Fernando Saavedra Peláez, saave@flacso.edu.mx, Profesor investigador, Facultad Latinoamericana de Ciencias Sociales sede México. 